\title{
openheart Myo-inositol for insulin resistance, metabolic syndrome, polycystic ovary syndrome and gestational diabetes
}

\author{
James J DiNicolantonio (D), James H O'Keefe (D)
}

To cite: DiNicolantonio JJ, H O'Keefe J. Myo-inositol for insulin resistance, metabolic syndrome, polycystic ovary syndrome and gestational diabetes. Open Heart 2022;9:e001989. doi:10.1136/ openhrt-2022-001989

Accepted 16 February 2022

Check for updates

(C) Author(s) (or their employer(s)) 2022. Re-use permitted under CC BY-NC. No commercial re-use. See rights and permissions. Published by BMJ.

Department of Preventive Cardiology, Saint Lukes Mid America Heart Institute, University of Missouri-Kansas City, Kansas City, Missouri, USA

Correspondence to Dr James J DiNicolantonio; jjdinicol@gmail.com
Myo-inositol, also known as inositol (cyclohexanehexol), is a cyclic carbohydrate with six hydroxyl groups. ${ }^{1}$ For a long time, it was considered a $\mathrm{B}$ vitamin (vitamin $\mathrm{B}_{8}$ ). However, it is not considered an essential nutrient because it is formed from glucose. Each kidney makes around $2 \mathrm{~g}$ of myo-inositol per day, and the average dietary intake is $0.5-1.0 \mathrm{~g} /$ day. $^{2}{ }^{3}$ The liver and brain also synthesise myo-inositol, although at much lower amounts compared with the kidneys. Importantly, however, in the brain, myoinositol levels reach concentrations 10-fold to 15-fold greater than that of blood, and there is limited uptake of myo-inositol from systemic circulation. ${ }^{3}$

Unfortunately, no requirement for dietary inositol in humans has been determined despite demonstration of signs of inositol deficiency in several animal species. ${ }^{4}$ Importantly, dietary caffeine intake (particularly coffee) increases the need for myo-inositol. ${ }^{5}$ Increasing age, antibiotic use, sugar and refined carbohydrate intake, sodium deficiency, insulin resistance, and type 1 and type 2 diabetes all increase the need for myo-inositol. ${ }^{6}$ Importantly, tissues highest in inositol, such as kidneys, brain and blood cells, are typically no longer consumed by humans. Furthermore, other foods that are high in inositol-forming substances, such as liver, grains, seeds and beans, are not readily consumed. Thus, from a dietary perspective, our intake of inositol is much lower compared with what humans would have consumed during Palaeolithic times.

We get myo-inositol in the diet either as inositol-containing phospholipids or phytic acid—which is inositol hexaphosphate (IP6). Bacterial phytases and phosphatases are primarily responsible for digesting dietary IP6 in the body and for releasing myo-inositol and phosphate. All living cells (animal, plant, bacteria and fungi) contain inositol phospholipids in their membranes either in their free form or bound as phospholipids or inositol phosphates. Phytic acid, the main storage form of phosphorus and source of inositol, is found in the bran of grains and seeds. Other inositol-containing foods include almonds, walnuts, Brazil nuts, oats, beans and peas, cantaloupe, and citrus fruits (except lemons). ${ }^{2}$ For example, $4 \mathrm{oz}$ of grapefruit juice contains around $470 \mathrm{mg}$ of myo-inositol. The average amount of myo-inositol in a 2000 kcal American diet is approximately $720 \mathrm{mg}$, of which $56 \%$ is lipid-bound. The intake of myo-inositol can be as low as $250 \mathrm{mg}$ or as high as $1650 \mathrm{mg} /$ day from a $2000 \mathrm{kcal}$ diet, depending on the foods that are consumed. ${ }^{78}$ In humans, myo-inositol doses of $18 \mathrm{~g}$ for 3 months or $4 \mathrm{~g}$ for 12 months have been noted to be safe and well tolerated. At higher doses, mild side effects can occur such as nausea, gas and diarrhoea.

Myo-inositol is synthesised from glucose, which eventually gets converted to free myoinositol. Free inositol can also be obtained by recycling inositol-triphosphate (IP3) and inositol-bisphosphate (IP2). Thus, taking myo-inositol likely spares IP3 and IP2. The biosynthesis of myo-inositol requires nicotinamide adenine dinucleotide+ and magnesium. Thus, a deficiency in either may lead to a deficiency in myo-inositol. ${ }^{39}$ Myo-inositol is the structural bases of many hormonal secondary messengers, particularly IP3 and phosphatidylinositol phosphate lipids $\left(\mathrm{PIP}_{2} /\right.$ $\mathrm{PIP}_{3}$ ) and inositol glycans. Myo-inositol exists in many phosphorylated forms, such as monophosphorylated forms (Ins-1-P, Ins3-P or Ins-4-P), pyrophosphate forms (PP$\mathrm{InsP}_{4}, \mathrm{PP}-\mathrm{InsP}_{5}, \mathrm{PP}_{2} \mathrm{INsP}_{3}$ or $(\mathrm{PP})_{2}$-InsP4) and a hexaphosphorylated form (IP6 or phytic acid) ${ }^{10}$ Furthermore, inositol is essential for normal cell growth and survival, development and function of peripheral nerves, osteogenesis and reproduction. ${ }^{71-19}$

Myo-inositol is a sugar alcohol or polyol, and it mediates signal transduction in response to 
several hormones, neurotransmitters and growth factors and participates in osmoregulation. ${ }^{20}$ There are eight other inositol isomers, all of which are formed from the epimerisation of myo-inositol. Myo-inositol also leads to the formation of inositol phosphates, phosphatidylinositol, phosphatidylinositol-phosphates, glycosyl-phosphatidylinositols, inositol-phosphoglycans, and inositol ethers and esters. ${ }^{1}$

Myo-inositol can either be present in free form or bound to phospholipids or inositol phosphate derivates. ${ }^{2}$ Additionally, myo-inositol is a structural component of the cell membrane as it is needed in the formation of phosphatidylinositol. ${ }^{1}$ Myo-inositol is also important for insulin signalling as it makes up many secondary messengers in response to insulin. Elevated glucose levels decrease the absorption and biosynthesis of myo-inositol and increase its degradation and urinary excretion. ${ }^{21}$ Insulin resistance and elevated glucose levels reduce inositol uptake into tissues. Myo-inositol levels are higher in tissues that use large amounts of glucose, such as the brain, heart and ovaries. D-chiro-inositol (DCI) is higher in tissues requiring glucose storage, such as liver and muscles. Insulin resistance impairs the conversion of myo-inositol to DCI in muscles, fat and liver. Increased urinary losses of myo-inositol have been consistently found in those with type 1 and type 2 diabetes. ${ }^{21}$

Once myo-inositol enters the cell, it is transformed into phosphatidyl-myo-inositol, which then converts to inositol-triphosphate acting as an intracellular second messenger for insulin, follicle-stimulating hormone (FSH) and thyroid-stimulating hormone (TSH).$^{22}$ Myoinositol and DCI are the two main inositol stereoisomers in the human body. DCI is formed from the conversion of myo-inositol via an insulin-dependent mechanism. ${ }^{23}$

\section{INOSITOL TRANSPORT AND EXCRETION}

Myo-inositol is actively transported by intestinal cells in a Nat-dependent manner. ${ }^{24}$ Virtually all of the free myoinositol $(99.8 \%)$ is absorbed from the human gastrointestinal tract through this active transport system involving the $\mathrm{Na}^{+} / \mathrm{K}^{+}$-ATPase. ${ }^{25}$ Thus, sodium helps with absorption of myo-inositol in the intestine and its reabsorption in the kidneys. ${ }^{1}$ Additionally, sodium moves myoinositol into tissues cells. The major transport system for myo-inositol uptake into mammalian cells is a saturable carrier-mediated active process driven by energy from the sodium gradient. Indeed, two sodium ions transport one myo-inositol into the brain and peripheral tissues via sodium-dependent myoinositol transporter 1 (SMIT1) and sodium-dependent myoinositol transporter 2 (SMIT2) ${ }^{26}$ Inositol can be transported from extracellular fluid via three transporters: SMIT1, SMIT2 and H+-myoinositol transporter, which cotransports myo-inositol with $\mathrm{H}+$. SMIT1 and SMIT2 cotransport two sodium ions to generate enough energy to actively transport myoinositol into the cell. SMIT1 and SMIT2 help drive myoinositol into the brain, and the intestinal absorption and reabsorption in the kidney depends on SMIT2, which is inhibited by glucose. ${ }^{7}$ SGLT1 $/ 2$ inhibitors reduce myoinositol absorption/reabsorption. Galactose and glucose reduce myo-inositol uptake and incorporation into phospholipids. This occurs even under physiological concentrations of glucose. ${ }^{27}$ Elevated blood glucose depletes myo-inositol levels in nervous tissue due to a competitive inhibition of the sodium-dependent myo-inositol uptake. ${ }^{10}$ Glucose and other sugars reduce the intestinal absorption and kidney reabsorption of myo-inositol. ${ }^{1}$ Manganese helps to incorporate free inositol into the phosphoinositides and the formation of phosphatidylinositol. $^{28}$

In rats, myo-inositol accumulates rapidly (within 1 hour) after absorption in large amounts in the thyroid, sex glands and reproductive fluids. Other tissues, including the pituitary, prostate, liver and spleen concentrate myoinositol. Importantly, all organs of the male reproductive tract, except for the testis, have myo-inositol levels that are 10-30 times that of blood. This is likely because the testis can endogenously synthesise inositol, as can the brain, kidney and liver. About half of the free inositol in rabbit brain is from endogenous production, the other half coming from blood. The kidneys are the main organ to catabolise inositol into $\mathrm{CO}_{2}$. However, the kidneys may metabolise inositol into other metabolites that are more readily able to cross the blood-brain barrier. ${ }^{7}$

The organs of the male reproductive tract (and epididymal, vesicular and prostatic fluids) are rich in free myoinositol. Moreover, high levels of myo-inositol have been measured in mammalian semen, suggesting its importance in male reproductive health. ${ }^{1}$ Unbound free myoinositol levels are high in the brain, cerebrospinal fluid, choroid plexus, small intestine and kidney, whereas myoinositol is primarily in its phospholipid-bound form in the liver, skeletal muscle and heart. Myo-inositol is also an important constituent of breast milk. The organs that concentrate myo-inositol the most are the kidneys, organs of the reproductive tract and the brain. Except for the brain, myo-inositol tissue content is highly dependent on dietary inositol, suggesting that a lack of inositol in the diet could lead to suboptimal tissue levels. ${ }^{129}$

\section{MYO-INOSITOL AND DCI FOR POLYCYSTIC OVARY SYNDROME (PCOS)}

PCOS affects $5 \%-21 \%$ of women during their reproductive life. ${ }^{23}$ Insulin resistance is a frequent finding in patients with PCOS, regardless of body mass index (BMI). Indeed, around $70 \%-80 \%$ of women with PCOS and central obesity and $15 \%-30 \%$ of lean women with PCOS have insulin resistance and compensatory hyperinsulinaemia. The hyperinsulinaemia can increase ovarian androgen synthesis via amplified luteinising hormone (LH) secretion, which also affects ovarian stimulation.

Insulin uses inositol phosphoglycans (IPGs) as secondary messengers, which control the oxidative and non-oxidative metabolisms of glucose as well as 
the uptake of glucose by GLUT4. ${ }^{7}$ Additionally, other hormones such as TSH and FSH use IPGs as secondary messengers. ${ }^{23}$ Both myo-inositol and DCI are involved in the intracellular transmission of insulin's metabolic signal and are also important for the oxidative use of glucose and its storage as glycogen. ${ }^{730}$

The epimerase that converts myo-inositol to DCI is insulin dependent, and this conversion is reduced in insulin resistance tissues. There is a reduction in urinary DCI and an increase in urinary myo-inositol excretion in humans and animals with type 2 diabetes. ${ }^{31}{ }^{32}$ Patients without PCOS undergoing a glucose tolerance test have a threefold lower release of DCI than controls. ${ }^{33} 34$ Thus, a deficiency in myo-inositol, or an impairment in the function/expression of the enzyme that converts myoinositol to DCI, can lead to insulin resistance (which can further lead to a deficiency in DCI since the epimerase that converts myo-inositol to DCI is insulin sensitive) ${ }^{23}$

DCI at $1200 \mathrm{mg}$ /day given to obese patients with PCOS for 8 weeks significantly reduced insulin area under the curve after an oral glucose tolerance test by $62 \%$ and free testosterone levels by $55 \% .{ }^{35}$ Diastolic and systolic blood pressure significantly decreased by $4 \mathrm{~mm} \mathrm{Hg}$, and triglyceride concentrations decreased from $184 \mathrm{mg} / \mathrm{dL}$ to $110 \mathrm{mg} / \mathrm{dL}$ compared with placebo. Lastly, ovulation was restored in $86 \%$ of patients receiving DCI vs only $27 \%$ on the placebo group. Lean patients with PCOS also benefit when myo-inositol is combined with DCI regarding restoring the regularity of their menstrual cycle. ${ }^{36}$

Unfer and colleagues looked at 12 studies and found that myo-inositol (with or without DCI) improves oocyte maturation, pregnancy rates and hormonal parameters such as LH, LH:FSH ratio, testosterone, androstenedione, insulinaemia and Homeostatic Model Assessment (HOMA) index in patients with PCOS. ${ }^{37}$ The glucose:insulin ratio, total cholesterol and high-density lipoprotein (HDL) concentrations also improved.

In a 12-week randomised controlled study in 50 overweight women with PCOS, $2 \mathrm{~g}$ of myo-inositol (with $200 \mathrm{mcg}$ of folic acid) in the morning led to significant reductions in plasma $\mathrm{LH}$, prolactin, testosterone, insulin and LH:FSH ratio and an improvement in insulin sensitivity. ${ }^{38}$ Delivery rate also significantly improved with myo-inositol, and menstrual cyclicity was restored in all amenorrheic and oligomenorrheic subjects. No changes in menstrual cyclicity occurred in the patients treated with folic acid alone. Additionally, Kamenov et al found that $2 \mathrm{~g}$ /day of myo-inositol improved ovulation leading to higher rates of pregnancy and delivery. ${ }^{39}$ A reduction in BMI and an improvement in insulin resistance were also noted. This study also found that around $61.7 \%$ of patients with PCOS ovulated after taking myo-inositol, and in those who were resistant, once clomiphene was added, $72.2 \%$ ovulated. Several meta-analyses of randomised controlled trials have shown that myo-inositol monotherapy, and when combined with DCI, is effective in patients with PCOS. ${ }^{40-43}$ However, myo-inositol is better tolerated than metformin, making it more acceptable for
PCOS women. ${ }^{44}$ Another meta-analysis concluded that myo-inositol is beneficial in PCOS and non-PCOS women undergoing in vitro fertilisation. ${ }^{42}$

\section{INOSITOLS IN INSULIN SIGNALLING AND GLUCOSE METABOLISM}

Much of the benefits of high-fibre diets in regard to cancer reduction and improvements in glucose metabolism are thought to be due to the IP6 content. ${ }^{45}$ Insulin-resistant states lead to increased urinary losses of myoinositol primarily due to the glucose-mediated inhibition of myoinositol reabsorption by the kidneys. ${ }^{3}$ A reduction in myoinositol decreases DCI, in which a deficiency in both contributes to insulin resistance in skeletal muscle, liver and fat cells. Myo-inositol is involved in fertility, oogenesis, embryogenesis, regenerative processes (transcription control, mRNA export and DNA repair) and fat metabolism. ${ }^{3}$

Myo-inositol inhibits duodenal glucose absorption and reduces blood glucoses rises via a competitive affinity for the same transporter system. ${ }^{46}$ Ironically, however, myo-inositol improves muscle glucose uptake. Glucose also impairs cellular uptake of myo-inositol. Sodiumglucose transport inhibitors prevent glucose and inositol uptake. ${ }^{3}$ Inositol is transported into the cell by sodium ion-coupled transporters, and glucose can competitively inhibit the uptake of inositol via this pathway. ${ }^{10} 47$ This is likely why diabetic nerves are depleted in inositol. Glucose can also deplete myoinositol via activation of the glucose-sorbitol pathway, whereby glucose is converted to sorbitol by aldose reductase and then to fructose, which is increased in states of elevated glucose. The rise in sorbitol elevates intracellular osmolarity, which inhibits the uptake of other osmolytes, including myo-inositol, by downregulating the expression of their carriers. In fact, aldose reductase inhibition restores myoinositol levels by reducing sorbitol levels. ${ }^{3}$ Thus, states of elevated glucose increase inositol needs.

Abnormalities in plasma and urine myo-inositol/ DCI may be an early marker of insulin resistance and type 2 diabetes. ${ }^{48}$ Furthermore, a lack of myo-inositol worsens insulin resistance, impairs antioxidant defenses and increases oxidative glycation stress. Phospholipase C releases IPGs, containing either myo-inositol or DCI, both of which have insulin-mimetic activity and act as second messengers downstream of insulin receptors. Inositol compounds also reduce hyperglycaemia in a dose-dependent fashion and promote glycogen stores in muscle. $^{3}$

The conversion of myo-inositol to DCI is severely impaired with insulin resistance in insulin-sensitive tissues (muscle, fat and liver). Thus, a low DCI:myo-inositol ratio may represent a measure of insulin resistance. Indeed, low DCI levels are typical in the urine and muscle tissue of patients with type 2 diabetes. ${ }^{3149}$ A lack of DCI in the urine is related with insulin resistance in both patients with type 2 and healthy controls. ${ }^{50}$ A lack of myo-inositol 
will reduce DCI levels and worsen insulin resistance. Plasma and tissue levels of DCI and IPGs are reduced in patients with PCOS with insulin resistance and in patients with type 2 diabetes. ${ }^{33}$

A paradox exists in patients with PCOS, whereby the ovaries do not become insulin resistant, even though these patients have insulin resistance and issues with ovulation. In fact, there is a greater conversion of myo-inositol to DCI in the ovaries, which leads to low myo-inositol in the ovary and high DCI, leading to biological dysfunctions. ${ }^{51}$ This is why combining both myo-inositol (to fix the low myo-inositol in the ovary) and DCI (to fix the low levels of DCI in the liver, skeletal muscle and fat) has synergistic benefits. Indeed, impairment in ovary function in patients with PCOS is associated with an increase in the DCI:myo-inositol ratio. ${ }^{52}$

In sites of diabetic microvascular complications (kidney, sciatic, nerve, retina and lens), a concomitant depletion of intracellular myo-inositol and accumulation of intracellular sorbitol is commonly observed. ${ }^{7}$ In diabetes, there is reduced cellular myo-inositol uptake, altered myo-inositol biosynthesis, enhanced efflux due to sorbitol intracellular accumulation and increased degradation leading to myo-inositol depletion. ${ }^{7}$ The competition between myo-inositol and glucose for myoinositol transporters occurs due to the similarity in their structure. ${ }^{47}$

In the testes of diabetic animals, there is a significant $50 \%$ reduction in the activity of 1-D-myo-inositol-phosphate synthase, the enzyme that regulates the first step in myoinositol biosynthesis. ${ }^{7}$ However, other tissues (kidney, brain and nerves) do not show a reduction in enzyme activity. There is an increase in the enzyme that breaks down myo-inositol in the kidney of diabetic, insulin resistant, and hypertensive rats and mice. ${ }^{7}$

\section{How states of insulin resistance deplete myo-inositol}

- Reduced synthesis (reduced biosynthesis via decreased enzyme function).

- Increased breakdown (upregulation in the enzyme that breaks down myo-inositol).

- Decreased penetration into cells (competition with glucose).

- Greater cellular release/reduced cellular uptake (sorbitol competition).

- Greater loss in the urine (glucose competes for myoinositol reabsorption in the kidneys).

Myo-inositol at $2 \mathrm{~g}$ two times per day reduces the incidence of gestational diabetes by $65.0 \%-87.3 \% .{ }^{53} 54$ These studies also noted reduced requirements for insulin, delivery at a later gestational age and fewer episodes of neonatal hypoglycaemia. In a randomised, placebocontrolled study, 80 women were placed on either myoinositol $2 \mathrm{~g}$ two times per day or placebo. The group treated with myo-inositol had significant improvements in diastolic blood pressure (-11\%), HOMA index value $(-75 \%)$, serum triglycerides $(-20 \%)$ and HDL $(+22 \%){ }^{55}$ In 69 women with gestational diabetes, $2 \mathrm{~g}$ of myo-inositol two times per day significantly improved fasting glucose and insulin and Homeostatic Model Assessment for Insulin Resistance (HOMA-IR) value compared with control. ${ }^{56}$ Furthermore, adiponectin increased in the myo-inositol group but decreased in the control group. ${ }^{756}$ In 80 postmenopausal women with metabolic syndrome, $2 \mathrm{~g}$ /day of myo-inositol improved blood glucose, insulin, HOMA-IR value, triglycerides, total and HDL cholesterol and blood pressure compared with placebo. ${ }^{57}$ Additionally, there was a significant improvement in BMI and waist circumference in the myo-inositol group versus baseline after 12 months. After taking myo-inositol, eight women (20\%) no longer had metabolic syndrome compared with only one in the control group.

...inositol is involved in many cell functions, especially as a precursor of phosphatidylinositol and phosphoinositides. Since the Km for the biosynthesis of phosphatidylinositol from myo-inositol is relatively high... a depletion of intracellular myo-inositol could have a negative impact on the synthesis and availability of phosphatidylinositol and phosphatidylinositol phosphates in cells. ${ }^{7}$

Altered phosphatidylinositol turnover has been associated with myo-inositol deficiency in the sciatic nerve of diabetic rats. ${ }^{58}$ Altered Na/K-ATPase activity may be a direct consequence of intracellular myo-inositol deficiency and a potential reason for diabetic microvascular complications. Indeed, altered $\mathrm{Na} / \mathrm{K}-\mathrm{ATPase}$ activity impairs nerve conductivity and may cause diabetic neuropathy (axonal degeneration and demyelination) through inhibition of cell growth, transformation and differentiation. A depletion of myo-inositol, caused by elevated glucose levels, may be directly responsible for diabetic glomerulosclerosis and proteinuria as well as tubulointerstitial fibrosis, which would reduce the reabsorption of inositol and other nutrients (electrolytes and amino acids) back into the body. ${ }^{7}$

...the myo-inositol depletion observed under hyperglycemic conditions in insulin insensitive tissues seems to contribute to the development of diabetic microvascular complications, together with the four major and more recognized pathways, namely: increased Advanced Glycation End products (AGEs) formation, activation of protein kinase $\mathrm{C}$ (PKC), increased hexosamine and sorbitol pathways. ${ }^{7}$

Consequences of myo-inositol deficiency ${ }^{7}$

- Altered Na-K-ATPase activity.

- Diabetic neuropathy, nephropathy and retinopathy.

- Diabetic nephropathy.

- Worsening of insulin resistance.

- Elevated fasting and postprandial glucose and insulin levels.

Individuals with type 2 diabetes have a decreased urinary excretion of chiro-inositol and a 10-fold higher urinary excretion of myo-inositol compared with healthy people. ${ }^{31}$ Elevated urinary myo-inositol levels in 
individuals with type 2 diabetes have been known since 1859. In monkeys, there is a progressive increase in myoinositol levels in the urine when going from normal to obese non-diabetic to diabetic, which correlates with the severity of insulin resistance. ${ }^{7}$ An increased myoinositol:DCI ratio in the urine has been proposed as an index of insulin resistance in humans. A decrease of DCI in insulin target tissues could reduce insulin signal transduction and further contribute to insulin resistance in those tissues. There are also depleted plasma levels of the DCI in patients with PCOS. ${ }^{7}$

In autopsy and biopsy studies of the muscles of individuals with type 2 diabetes, there is an increased myo-inositol:DCI ratio. Furthermore, chiro-inositol is decreased in the muscle, urine and hemodialysate of individuals with type 2 diabetes versus controls. ${ }^{49}$ There is a fourfold to fivefold decrease in the conversion of myoinositol to DCI in type 2 diabetic rats due to a twofold to threefold decrease in epimerase activity. The reduction in epimerase activity is likely caused by myo-inositol deficiency, insulin insensitivity or a combination of both. ${ }^{7}$

Insulin resistance and diabetes lead to

- Abnormally low levels of DCI in urine, plasma and insulin-sensitive tissues (liver, muscle and fat).

- Excessive urinary excretion of myo-inositol.

- Intracellular myo-inositol deficiency in insulininsensitive tissues (kidney, ovary, sciatic nerve, lens and retina).

\section{DIABETES, GESTATIONAL DIABETES AND METABOLIC SYNDROME}

In gestational diabetes, myo-inositol at $2 \mathrm{~g}$ two times per day for 8 weeks improves fasting insulin and glucose levels. ${ }^{56}$ In pregnant women with a family history of type 2 diabetes, myo-inositol at $2 \mathrm{~g}$ two times per day throughout pregnancy significantly reduces fasting and 1-hour glucose levels after an oral glucose tolerance test and reduces the incidence of gestational diabetes by $40 \%$ (6.0\% vs $15.3 \%$ in the placebo group).$^{53}$ Improvements were also noted in fetal macrosomia and high mean fetal weight. Blood pressure, total and low-density lipoprotein cholesterol, and serum triglycerides were also reduced and HDL was increased. ${ }^{753}$

Myo-inositol lowers serum insulin and improves insulin resistance approximately twofold better compared with pioglitazone or metformin, which are considered the gold standard medications in those with impaired glucose tolerance. ${ }^{7}$ Animal studies confirm that high doses of myo-inositol, given acutely or chronically, reduce blood glucose levels to an oral glucose load via improvements in peripheral insulin sensitivity by an enhanced GLUT-4 translocation to the plasma membrane in response to hyperglycaemia in skeletal muscle. ${ }^{7}$

\section{DIABETIC COMPLICATIONS}

Diabetic tissues, such as the kidney, sciatic nerve, lens and retina, are depleted in myo-inositol. Thus, myo-inositol supplementation may help to prevent or delay the development of diabetic neuropathy, nephropathy and/or retinopathy. ${ }^{7}$

\section{NEUROPATHY, NEPHROPATHY AND CATARACTS}

Myo-inositol supplementation in diabetic rats restores depleted myo-inositol intracellular levels and reduces or prevents impaired motor nerve conduction velocity despite persistent hyperglycaemic and elevated nerve intracellular levels of sorbitol and fructose. ${ }^{59}$ Myo-inositol may also benefit motor nerve conduction velocity in diabetics. ${ }^{60}$ Myo-inositol also prevents defects in axonal transport and motor nerve conduction velocity in diabetic rats. ${ }^{61}$ In patients with symptomatic distal symmetrical diabetic polyneuropathy, a high myo-inositol diet significantly increases median sensory and the sural sensory nerve conduction velocities. ${ }^{7}$ Myo-inositol supplementation reverses elevated glucose-induced reductions in cell proliferation and increased procollagen transcription and secretion in proximal tubule cells of the kidney. ${ }^{62}$ Myo-inositol supplementation can restore depleted intracellular myo-inositol levels in the lens of diabetic rats and reduce cataract formation. ${ }^{63}$ Furthermore, one review paper concluded, 'Correction of myo-inositol deficiency by dietary myo-inositol supplement prevented or delayed the development of some microvascular complications of diabetes in the motor nerves and lens in type 1 diabetes animal models'. ${ }^{7}$

Factors that deplete inositol include: $:^{79645}$

- Insulin resistance

- Elevated glucose levels

- Low sodium intake

- Caffeine (coffee, tea, etc)

- Kidney damage

- Intestinal damage

- Lithium

- Valproic acid

- Magnesium deficiency

\section{CONCLUSION}

Myo-inositol should be considered in patients with insulin resistance, metabolic syndrome, type 1 diabetes, type 2 diabetes, PCOS and those with or at risk of gestational diabetes. Elevated levels of glucose reduce myo-inositol levels in tissues and increase its breakdown and elimination via the kidneys. Myo-inositol has been used safely for decades in many studies in those with insulin resistance and PCOS.

Contributors All authors contributed to the final manuscript.

Funding The authors have not declared a specific grant for this research from any funding agency in the public, commercial or not-for-profit sectors.

Competing interests JJD is director of scientific affairs at AIDP. JJD is affiliated with companies that sell myo-inositol. JHO owns a supplement company.

Patient consent for publication Not applicable.

Ethics approval This study does not involve human participants. 
Provenance and peer review Not commissioned; internally peer reviewed.

Open access This is an open access article distributed in accordance with the Creative Commons Attribution Non Commercial (CC BY-NC 4.0) license, which permits others to distribute, remix, adapt, build upon this work non-commercially, and license their derivative works on different terms, provided the original work is properly cited, appropriate credit is given, any changes made indicated, and the use is non-commercial. See: http://creativecommons.org/licenses/by-nc/4.0/.

\section{ORCID iDs}

James J DiNicolantonio http://orcid.org/0000-0002-7888-1528

James H O'Keefe http://orcid.org/0000-0002-3376-5822

\section{REFERENCES}

1 Bizzarri M, Fuso A, Dinicola S, et al. Pharmacodynamics and pharmacokinetics of inositol(s) in health and disease. Expert Opin Drug Metab Toxicol 2016;12:1181-96.

2 Corrado F, Santamaria A. Chapter 19 - Myoinositol Supplementation on Insulin Resistance in Gestational Diabetes. In: Watson RR, Dokken BB, eds. Glucose intake and utilization in pre-diabetes and diabetes. Boston: Academic Press, 2015: 229-34.

3 Bevilacqua A, Bizzarri M. Inositols in insulin signaling and glucose metabolism. Int J Endocrinol 2018;2018:1968450

4 Chu SH, Hegsted DM. Myo-inositol deficiency in gerbils: changes in phospholipid composition of intestinal microsomes. J Nutr 1980;110:1217-23.

5 De Grazia S, Carlomagno G, Unfer V, et al. Myo-inositol soft gel capsules may prevent the risk of coffee-induced neural tube defects. Expert Opin Drug Deliv 2012;9:1033-9.

6 Daughaday $\mathrm{WH}$, Larner J. The renal excretion of inositol in normal and diabetic human beings. J Clin Invest 1954;33:326-32.

7 Croze ML, Soulage CO. Potential role and therapeutic interests of myo-inositol in metabolic diseases. Biochimie 2013;95:1811-27.

8 Clements RS, Darnell B. Myo-inositol content of common foods: development of a high-myo-inositol diet. Am J Clin Nutr 1980;33:1954-67.

9 Eisenberg F, Parthasarathy R. Measurement of biosynthesis of myo-inositol from glucose 6-phosphate. Methods Enzymo 1987;141:127-43.

10 Greene DA, Lattimer SA. Sodium- and energy-dependent uptake of myo-inositol by rabbit peripheral nerve. competitive inhibition by glucose and lack of an insulin effect. J Clin Invest 1982;70:1009-18.

11 Eagle H, OYAMA VI, LEVY M, et al. Myo-Inositol as an essential growth factor for normal and malignant human cells in tissue culture. J Biol Chem 1957;226:191-205.

12 Chau JFL, Lee MK, Law JWS, et al. Sodium/Myo-Inositol cotransporter-1 is essential for the development and function of the peripheral nerves. Faseb J 2005;19:1887-9.

13 Dai Z, Chung SK, Miao D, et al. Sodium/myo-inositol cotransporter 1 and myo-inositol are essential for osteogenesis and bone formation. J Bone Miner Res 2011;26:582-90.

14 Carlomagno G, Nordio M, Chiu TT, et al. Contribution of myo-inosito and melatonin to human reproduction. Eur J Obstet Gynecol Reprod Biol 2011;159:267-72.

15 Condorelli RA, La Vignera S, Bellanca S, et al. Myoinositol: does it improve sperm mitochondrial function and sperm motility? Urology 2012;79:1290-5.

16 Ciotta L, Stracquadanio M, Pagano I, et al. Effects of myo-inositol supplementation on oocyte's quality in PCOS patients: a double blind trial. Eur Rev Med Pharmacol Sci 2011;15:509-14.

17 Unfer V, Carlomagno G, Rizzo P, et al. Myo-inositol rather than Dchiro-inositol is able to improve oocyte quality in intracytoplasmic sperm injection cycles. A prospective, controlled, randomized trial. Eur Rev Med Pharmacol Sci 2011;15:452-7.

18 Condorelli RA, La Vignera S, Di Bari F, et al. Effects of myoinositol on sperm mitochondrial function in-vitro. Eur Rev Med Pharmacol Sci 2011;15:129-34.

19 Beemster P, Groenen P, Steegers-Theunissen R. Involvement of inositol in reproduction. Nutr Rev 2002;60:80-7.

20 Parthasarathy LK, Seelan RS, Tobias C, et al. Mammalian inosito 3-phosphate synthase: its role in the biosynthesis of brain inositol and its clinical use as a psychoactive agent. Subcell Biochem 2006;39:293-314

21 Dinicola S, Minini M, Unfer V, et al. Nutritional and acquired deficiencies in inositol bioavailability. correlations with metabolic disorders. Int J Mol Sci 2017;18. doi:10.3390/ijms18102187. [Epub ahead of print: 20 Oct 2017].

22 Thomas RM, Nechamen CA, Mazurkiewicz JE, et al. The adapter protein APPL1 links FSH receptor to inositol 1,4,5-trisphosphate production and is implicated in intracellular $\mathrm{Ca}(2+)$ mobilization. Endocrinology 2011;152:1691-701.

23 Genazzani AD. Inositol as putative integrative treatment for PCOS. Reprod Biomed Online 2016;33:770-80.

24 Caspary WF, Crane RK. Active transport of myo-inositol and its relation to the sugar transport system in hamster small intestine. Biochim Biophys Acta 1970;203:308-16.

25 Holub BJ. Metabolism and function of myo-inositol and inosito phospholipids. Annu Rev Nutr 1986;6:563-97.

26 Bourgeois F, Coady MJ, Lapointe J-Y. Determination of transport stoichiometry for two cation-coupled myo-inositol cotransporters: SMIT2 and HMIT. J Physiol 2005:563:333-43.

27 Kollros PE, Goldstein GW, Betz AL. Myo-inositol transport into endothelial cells derived from nervous system microvessels. Brain Res 1990;511:259-64.

28 Larner J, Price JD, Heimark D, et al. Isolation, structure, synthesis, and bioactivity of a novel putative insulin mediator. A galactosamine chiro-inositol pseudo-disaccharide Mn2+ chelate with insulin-like activity. J Med Chem 2003;46:3283-91.

29 Burton LE, Ray RE, Bradford JR, et al. Myo-Inositol metabolism in the neonatal and developing rat fed a myo-inositol-free diet. J Nutr 1976;106:1610-6.

30 Larner J, Brautigan DL, Thorner MO. D-chiro-inositol glycans in insulin signaling and insulin resistance. Mol Med 2010;16:543-52.

31 Kennington AS, Hill CR, Craig J, et al. Low urinary chiro-inosito excretion in non-insulin-dependent diabetes mellitus. $N$ Engl J Med 1990;323:373-8.

32 Ortmeyer HK, Bodkin NL, Lilley K, et al. Chiroinositol deficiency and insulin resistance. I. urinary excretion rate of chiroinositol is directly associated with insulin resistance in spontaneously diabetic rhesus monkeys. Endocrinology 1993;132:640-5.

33 Baillargeon J-P, Diamanti-Kandarakis E, Ostlund RE, et al. Altered D-chiro-inositol urinary clearance in women with polycystic ovary syndrome. Diabetes Care 2006;29:300-5.

34 Baillargeon J-P, Nestler JE, Ostlund RE, et al. Greek hyperinsulinemic women, with or without polycystic ovary syndrome, display altered inositols metabolism. Hum Reprod 2008;23:1439-46.

35 Nestler JE, Jakubowicz DJ, Reamer P, et al. Ovulatory and metabolic effects of D-chiro-inositol in the polycystic ovary syndrome. $N$ Engl J Med 1999;340:1314-20.

36 Le Donne M, Metro D, Alibrandi A, et al. Effects of three treatment modalities (diet, myoinositol or myoinositol associated with D-chiroinositol) on clinical and body composition outcomes in women with polycystic ovary syndrome. Eur Rev Med Pharmacol Sci 2019;23:2293-301

37 Unfer V, Carlomagno G, Dante G, et al. Effects of myo-inositol in women with PCOS: a systematic review of randomized controlled trials. Gynecol Endocrinol 2012;28:509-15.

38 Artini PG, Di Berardino OM, Papini F, et al. Endocrine and clinical effects of myo-inositol administration in polycystic ovary syndrome. A randomized study. Gynecol Endocrinol 2013:29:375-9.

39 Kamenov Z, Kolarov G, Gateva A, et al. Ovulation induction with myo-inositol alone and in combination with clomiphene citrate in polycystic ovarian syndrome patients with insulin resistance. Gynecol Endocrinol 2015;31:131-5.

40 Zhao $\mathrm{H}$, Xing $\mathrm{C}$, Zhang J, et al. Comparative efficacy of oral insulin sensitizers metformin, thiazolidinediones, inositol, and berberine in improving endocrine and metabolic profiles in women with PCOS: a network meta-analysis. Reprod Health 2021;18:171.

41 Azizi Kutenaei M, Hosseini Teshnizi S, Ghaemmaghami P, et al. The effects of myo-inositol vs. metformin on the ovarian function in the polycystic ovary syndrome: a systematic review and meta-analysis. Eur Rev Med Pharmacol Sci 2021;25:3105-15.

42 Laganà AS, Vitagliano A, Noventa $M$, et al. Myo-inositol supplementation reduces the amount of gonadotropins and length of ovarian stimulation in women undergoing IVF: a systematic review and meta-analysis of randomized controlled trials. Arch Gynecol Obstet 2018;298:675-84.

43 Unfer V, Facchinetti F, Orrù B, et al. Myo-inositol effects in women with PCOS: a meta-analysis of randomized controlled trials. Endocr Connect 2017:6:647-58.

44 Facchinetti F, Orrù B, Grandi G, et al. Short-term effects of metformin and myo-inositol in women with polycystic ovarian syndrome (PCOS): a meta-analysis of randomized clinical trials. Gynecol Endocrinol 2019;35:198-206.

45 Graf E, Eaton JW. Dietary suppression of colonic cancer. fiber or phytate? Cancer 1985;56:717-8.

46 Chukwuma Cl, Ibrahim MA, Islam MS. Myo-inositol inhibits intestinal glucose absorption and promotes muscle glucose uptake: a dual approach study. J Physiol Biochem 2016;72:791-801. 
47 Haneda M, Kikkawa R, Arimura T, et al. Glucose inhibits myo-inosito uptake and reduces myo-inositol content in cultured rat glomerular mesangial cells. Metabolism 1990;39:40-5.

48 Croze ML, Géloën A, Soulage CO. Abnormalities in myo-inositol metabolism associated with type 2 diabetes in mice fed a high-fat diet: benefits of a dietary myo-inositol supplementation. Br J Nutr 2015;113:1862-75.

49 Asplin I, Galasko G, Larner J. Chiro-inositol deficiency and insulin resistance: a comparison of the chiro-inositol- and the myo-inositolcontaining insulin mediators isolated from urine, hemodialysate, and muscle of control and type II diabetic subjects. Proc Natl Acad Sci U S A 1993;90:5924-8.

50 Suzuki S, Kawasaki H, Satoh Y, et al. Urinary chiro-inositol excretion is an index marker of insulin sensitivity in Japanese type II diabetes. Diabetes Care 1994;17:1465-8.

51 Unfer V, Carlomagno G, Papaleo E, et al. Hyperinsulinemia alters myoinositol to D-chiroinositol ratio in the follicular fluid of patients with PCOS. Reprod Sci 2014;21:854-8.

52 Carlomagno G, Unfer V, Roseff S. The D-chiro-inositol paradox in the ovary. Fertil Steril 2011;95:2515-6.

53 D'Anna R, Scilipoti A, Giordano D, et al. Myo-Inositol supplementation and onset of gestational diabetes mellitus in pregnant women with a family history of type 2 diabetes: a prospective, randomized, placebo-controlled study. Diabetes Care 2013;36:854-7.

54 Matarrelli B, Vitacolonna E, D'Angelo M, et al. Effect of dietary myoinositol supplementation in pregnancy on the incidence of maternal gestational diabetes mellitus and fetal outcomes: a randomized controlled trial. J Matern Fetal Neonatal Med 2013;26:967-72.

55 Giordano D, Corrado F, Santamaria A, et al. Effects of myo-inositol supplementation in postmenopausal women with metabolic syndrome: a perspective, randomized, placebo-controlled study. Menopause 2011;18:102-4.
56 Corrado F, D'Anna R, Di Vieste G, et al. The effect of myoinositol supplementation on insulin resistance in patients with gestational diabetes. Diabet Med 2011;28:972-5.

57 Santamaria A, Giordano D, Corrado F, et al. One-year effects of myoinositol supplementation in postmenopausal women with metabolic syndrome. Climacteric 2012;15:490-5.

58 Clements RS, Stockard CR. Abnormal sciatic nerve myo-inositol metabolism in the streptozotocin-diabetic rat: effect of insulin treatment. Diabetes 1980;29:227-35.

59 Greene DA, De Jesus PV, Winegrad Al. Effects of insulin and dietary myoinositol on impaired peripheral motor nerve conduction velocity in acute streptozotocin diabetes. J Clin Invest 1975;55:1326-36.

60 Salway JG, Whitehead L, Finnegan JA, et al. Effect of myo-inositol on peripheral-nerve function in diabetes. Lancet 1978;2:1282-4.

61 Mayer JH, Tomlinson DR. Prevention of defects of axonal transport and nerve conduction velocity by oral administration of myo-inositol or an aldose reductase inhibitor in streptozotocin-diabetic rats. Diabetologia 1983;25:433-8.

62 Ziyadeh FN, Simmons DA, Snipes ER, et al. Effect of myo-inositol on cell proliferation and collagen transcription and secretion in proximal tubule cells cultured in elevated glucose. J Am Soc Nephrol 1991;1:1220-9.

63 Beyer-Mears A, Bucci FA, Del Val M, et al. Dietary myo-inositol effect on sugar cataractogenesis. Pharmacology 1989;39:59-68.

64 Janiri L, D'Ambrosio F, Di Lorenzo C. Combined treatment of myo-inositol and D-chiro-inositol (80:1) as a therapeutic approach to restore inositol eumetabolism in patients with bipolar disorder taking lithium and valproic acid. Eur Rev Med Pharmacol Sci 2021;25:5483-9.

65 Gonzalez-Uarquin F, Rodehutscord M, Huber K. Myo-Inositol: its metabolism and potential implications for poultry nutrition-a review. Poult Sci 2020;99:893-905 